\title{
Distributed fiber optic sensors for monitoring reinforced concrete piles using Brillouin scattering
}

\author{
N. de Battista*a , C. Kechavarzi ${ }^{\mathrm{a}}$, K. Soga ${ }^{\mathrm{a}}$ \\ ${ }^{a}$ Centre for Smart Infrastructure and Construction, Engineering Department, Cambridge University, \\ Trumpington Street, Cambridge CB2 0QJ, UK
}

\begin{abstract}
In this paper we report on advances made in the installation and use of distributed fiber optic sensors to monitor reinforced concrete piles subjected to static load tests. Eight concrete test piles, at three construction sites in London, have recently been instrumented with embedded DFOS. The Brillouin optical time domain reflectometry (BOTDR) technique was used to measure the changes in internal strain and temperature of the piles, during concrete curing and during load testing. These data were used to assess the quality of the pile and derive the load capacity parameters to be used in the foundation design of tall buildings which are to be erected on these sites. The measurements obtained from the DFOS system agreed well with the measurements taken simultaneously using conventional point sensors embedded in the piles. Whereas the conventional sensors only provided measurements at a small number of locations within the piles, the DFOS system made it possible to record the complete strain / temperature profiles along the length of the piles.
\end{abstract}

Keywords: distributed fiber optic sensors, Brillouin scattering, Brillouin optical time domain reflectometry, BOTDR, pile foundations, pile testing, maintained load

\section{INTRODUCTION}

Fiber optic sensors are becoming increasingly popular for monitoring various types of civil infrastructure. Being immune to electromagnetic noise and exhibiting excellent long-term durability under severe environmental conditions, optical fibers offer a distinct advantage over traditional electrical sensors in this field. Moreover, distributed fiber optic sensors (DFOS) allow the acquisition of densely-spaced data over very long distances, with a single measuring device. In the Civil Engineering domain, where conventional sensors are almost exclusively point sensors, DFOS promises to be a revolutionary technology that brings unprecedented opportunity for scaling monitoring systems with relative ease.

Full-scale testing of pile foundations is one particular application where DFOS is expected to make a significant impact. Pile testing is carried out on a small number of working piles or trial piles in a construction site, in order to validate foundation design before a construction project commences ${ }^{1}$. Pile tests are also useful for quality control and continuous improvement of pile design and construction practices.

The maintained static vertical load test is one of the most common types of pile tests carried out in the UK. A controlled compressive or tensile load is applied to the test pile in stages, while the response of the pile is recorded. This entails measuring the vertical deflection at the top of the pile and the strain and relative displacement within the pile. The internal measurements are typically taken with embedded vibrating wire strain gauges (VWSG) and retrievable extensometers, placed at a few locations several meters apart along the pile.

These traditional instrumentation systems have been used for many years in the pile testing industry and they are considered to be reliable and robust. However they suffer from one major drawback: they consist of point sensors, and consequently they cannot give a complete picture of a pile's response along its whole depth. The readings from these sensors can be misleading if there happens to be a localized effect in the pile or in the ground close to the sensor. Conversely, point sensors cannot detect any localized effects which are not in their vicinity, and which might be important for the assessment of the pile performance. The only way that the complete strain and deflection profile along the pile can be obtained is by using a distributed measurement system. This is where DFOS can play an important role. 


\section{INSTRUMENTATION OF CONCRETE PILES WITH DISTRIBUTED FIBER OPTIC SENSORS}

Following several years of development and field trials, in 2015, the Centre for Smart Infrastructure and Construction (CSIC) at Cambridge University was tasked with instrumenting eight cast-in-situ reinforced concrete trial test piles, at three different sites in London, prior to the construction of tall buildings. The piles were $0.9 \mathrm{~m}$ in diameter and ranged in length between $25 \mathrm{~m}$ and $33.7 \mathrm{~m}$.

The DFOS instrumentation consisted of two types of single-mode fiber optic (FO) cables installed in parallel: a tightlybonded cable (Fujikura 4-core 9.5/125 JBT 03813 with reinforcing steel wires) and a loose-tube cable (Excel 4-core 9/125 OS1). Brillouin optical time domain reflectometry (BOTDR) was used to measure the concrete strain and temperature from these two cables respectively ${ }^{2}$. The two cables were installed as a pair, in U-shaped loops along the pile reinforcement cages, before the cages were inserted into the pile bore hole (Figure 1). Each loop consisted of a leg along one side of the cage, a wrap around half the cage circumference at the bottom, and a leg along the opposite side of the cage.

The FO cables were fixed to the pile reinforcement bars at each end of the pile, using plastic cable ties and slit rubber tubes (Figure 1c). Between attachment points, the cables were held close to the reinforcement using loose cable ties placed at intervals of 0.5 to $1 \mathrm{~m}$. The strain cable was pre-tensioned by hand between attachment points, before being fixed.

Each pile was instrumented with at least one FO cable loop. Two of the piles were instrumented with an additional FO loop perpendicular to the first, and another two piles were instrumented with an additional FO loop fixed to a bundle of reinforcement bars placed in the center of the pile. The individual temperature and strain cables exiting the top of a pile were connected together in series, either directly with fusion splices or by means of FC/APC connectors spliced to the cable ends. Thus the instrumentation of each pile consisted of a single DFOS circuit comprising temperature and strain cables, which could be interrogated simultaneously from a single channel of the optical analyzer.

In addition to the DFOS cables, all eight piles were instrumented with conventional instrumentation, consisting of embedded VWSG pairs and retrievable extensometers, at up to seven locations along the pile. Strings of electrical temperature sensors at $30 \mathrm{~cm}$ intervals were also embedded within the piles to measure the concrete curing temperature along three sides of the pile. These three tried and tested instrumentation systems were used as benchmarks to assess the accuracy of the DFOS data.

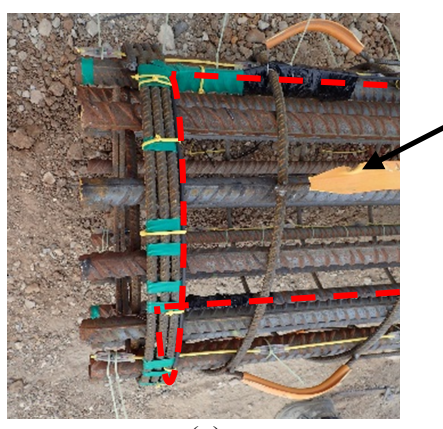

(a)

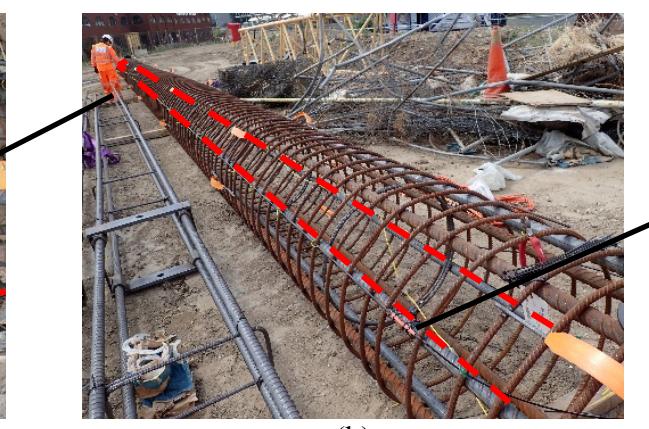

(b)

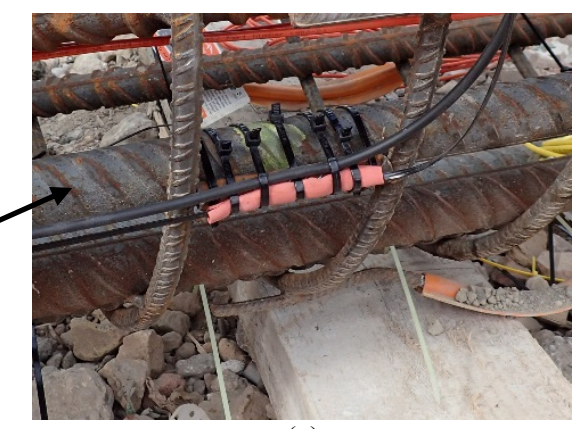

(c)

Figure 1. Details of the fiber optic cable installation on one of the pile reinforcement cages (cables highlighted with dashed red lines): (a) cables wrapped around the bottom of the cage; (b) layout of the cable loop; (c) cables attached to the reinforcement bars using cable ties and a slit rubber tube.

\section{MONITORING RESULTS}

The testing of the piles consisted of two phases: first to examine their geometric accuracy and homogeneity (pile integrity) from the concrete curing temperature profile following construction, and second to estimate the pile capacity and stiffness during maintained static load testing. In this section, a selection of the results obtained from the DFOS system during these two test phases of one of the piles is presented. 
The pile in question was $25 \mathrm{~m}$ long and the reinforcement consisted of a single $20 \mathrm{~m}$-long cage and a $25 \mathrm{~m}$-long central bar bundle. Two FO loops were installed in this pile, one on the cage and another on the bar bundle. Thus the temperature and strain were measured from the DFOS system along two opposite sides on the perimeter of the pile and also along the center of the pile. A Yokogawa AQ8603 BOTDR analyzer was used to take readings from the DFOS in this pile. The sampling resolution was set at $0.1 \mathrm{~m}$ and a spatial resolution of $1 \mathrm{~m}$ was used, with each measurement consisting of $2^{16}$ averages.

\subsection{Temperature monitoring during concrete curing}

The concrete curing temperature profiles of the pile were recorded with the DFOS system at 30 minute intervals, starting from 4 hours after the pile was completed. Figure 2 a shows the change in temperature along the center of the pile, as the concrete temperature increased gradually, until it reached a maximum temperature increase of $25.8^{\circ} \mathrm{C}$. This occurred 18 hours after the pile was constructed. The data from the embedded temperature sensors followed a very similar temperature profile as that recorded with the DFOS system.

A section of the pile from around 5 to $15 \mathrm{~m}$ depth attained consistently higher temperatures than the rest of the pile, during the concrete curing stage. Evidently the heat generated by the concrete hydration was dissipated slower into the ground at this section than it was along the rest of the pile. This indicates that the pile had a larger diameter at this section, implying an overbreak (the pile bore being excavated wider than intended). The section in question happened to coincide with the Alluvium and River Terrace Deposits strata of the ground.

\subsection{Strain monitoring during static load testing}

During the maintained static load test, the pile was loaded vertically in compression over two cycles, from a hydraulic loading rig directly above the pile head. Each cycle consisted of a number of load stages, with the applied load increasing gradually up to the maximum cycle load and then decreasing gradually back to zero load. At each stage, the load was maintained for a minimum of one hour, during which at least one strain and temperature measurement was taken with the DFOS system. The temperature readings were used to compensate the strain readings for thermal effects, in order to obtain a value of true strain. The applied load was measured with a load cell between the hydraulic rig and the pile head, and the settlement of the pile was measured using displacement transducers located around the pile head.

Figure $2 \mathrm{~b}$ shows the (thermally compensated) strain change profile measured along the center of the pile with the DFOS system, for each of the loading stages of the second cycle. This loading cycle lasted for 28 hours and consisted of seven load stages, starting from 7.7 MN up to $20 \mathrm{MN}$. A maximum compressive strain change of $772 \mu \varepsilon$ was recorded at a depth of around $5 \mathrm{~m}$ when the pile was subjected to the highest load. The strain readings obtained from VWSGs embedded at five levels within the pile agreed well with the DFOS strain measurements at those locations.

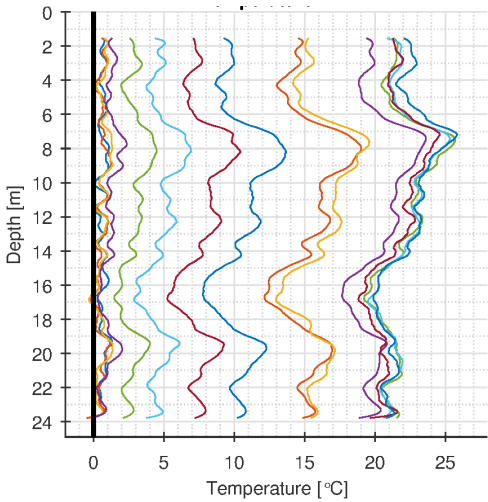

(a)

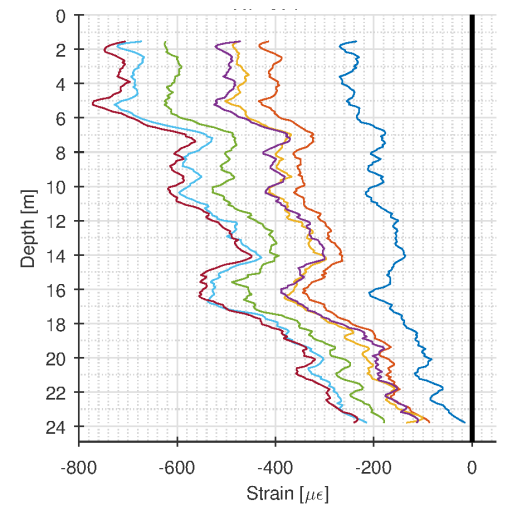

(b)

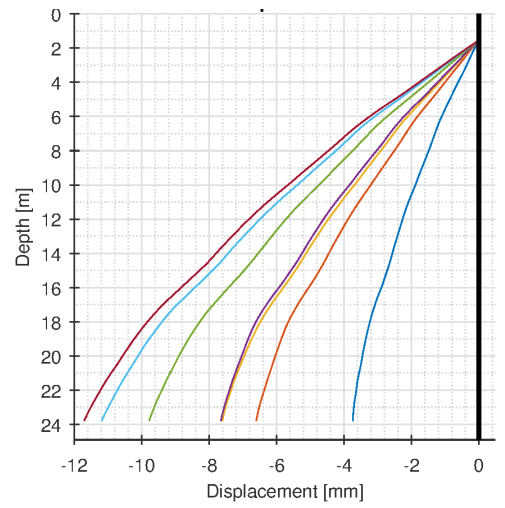

(c)

Figure 2. Data obtained from a DFOS system embedded in a reinforced concrete test pile: (a) Hourly concrete curing temperature change, starting from 4 hours after the pile was completed, until the maximum temperature was recorded 18 hours after pile completion; (b) strain change and (c) internal displacement when the pile was loaded incrementally in vertical compression with loads of 7.7 MN, 11.4 MN, 13.3 MN, 15.2 MN, 17.1 MN, 18.9 MN and 20.0 MN (increasing load corresponding to increasing negative strain / displacement). Negative strain and displacement indicates compression. 
For any given load, the strain in the pile would be expected to decrease gradually with depth, as the load gets transferred to the ground via shaft friction. This is generally the case with the strain profiles shown in Figure $2 \mathrm{~b}$. However, at the section between 5 and $15 \mathrm{~m}$, the strain change decreases bodily. This is likely due to the pile having a larger diameter in this section, resulting in a smaller strain for a given load. This reinforces the suspicion of an overbreak in the Alluvium and River Terrace Deposits strata, as was indicated by the curing temperature data.

The strain change profiles were integrated from top to bottom in order to obtain an estimate of the internal displacement (shortening) of each point along the pile, relative to the top of the pile. This is shown in Figure 2c for the same load cycles mentioned above. Once again, this data was in good agreement with the readings taken from retrievable extensometers placed at five levels within the pile.

\section{CONCLUSION}

Distributed fiber optic sensor (DFOS) technology promises to be a very useful tool in civil infrastructure monitoring. Not only are DFOS immune to electromagnetic noise and long-lasting when embedded in structures, they also allow the acquisition of data over very long distances, with unprecedented spatial density, along a fiber optic cable. In this paper we have reported on the use of DFOS with BOTDR interrogation to monitor reinforced concrete piles.

Eight concrete test piles were instrumented with embedded DFOS, at three construction sites in London. The purpose of the piles was to measure how they reacted to applied controlled loads, from which the Engineers could estimate the pilesoil interface properties for the foundation design of new buildings on these sites. Some of the results obtained from the monitoring of one of these piles were presented in this paper.

The DFOS system in each pile was first used to monitor the temperature profiles of the pile for several hours while the concrete was curing. From the temperature data it was possible to infer the integrity of the pile and assess its geometrical accuracy. The example shown in the paper indicated the presence of an overbreak in a section of the pile in question.

Subsequently the DFOS systems were used to measure the strain change along the depth of the piles during maintained static load testing. This data clearly showed how, for any given load, the strain decreased with depth along the pile, as the load was transferred to the ground via shaft friction. Once again, the example shown here confirmed the likely presence of an overbreak at the same section of the pile. Furthermore, the strain data were used to estimate the internal shortening at each point of the pile under the various applied loads.

The temperature, strain and displacement data obtained from the DFOS measurements agreed well with readings taken at the same time by conventional embedded electrical sensors. The latter consisted of strings of closely-spaced temperature sensors, vibrating wire strain gauges and retrievable extensometers. What these eight pile monitoring projects have shown is that, from a single DFOS system, one is able to obtain the data that would traditionally require three different instrumentation systems. In addition, whereas traditional sensors can only provide point readings at a small number of locations along a pile, with a DFOS system one is able to measure the complete temperature and strain profiles along the whole pile.

\section{ACKNOWLEDGEMENTS}

The authors would like to acknowledge the contribution of (listed in alphabetical order): Hyungjoon Seo, Jason Shardelow, Peter Knott, Yi Rui and Zili Li from CSIC; Duncan Nicholson, Landi Proctor, Stuart Pennington and Vivien Kwan from Ove Arup \& Partners Ltd.

\section{REFERENCES}

[1] Federation of Piling Specialists., Handbook on pile load testing, Federation of Piling Specialists, Beckenham, Kent, UK (2006).

[2] Kurashima, T., Horiguchi, T., Izumita, H., Furukawa, S.., Koyamada, Y., "Brillouin optical-fiber time domain reflectometry," IEICE Trans. Cummunications E76-B(4), 382-390 (1993). 